\title{
MICROWAVE ASSISTED SYNTHESIS OF SOME 2,4 DIHYDROXY 1,8-NAPHTHYRIDINES AND THEIR DERIVATIVES DEVOID OF SOLVENT AND CATALYST
}

\author{
Thirumala Chary Maringanti ${ }^{1{ }^{1 *}}$, Laxminarayana Eppakayala ${ }^{2}$, Shiva Shankar Sripelly ${ }^{1}$ and \\ Narender.Atmakuri ${ }^{1}$ \\ 1. Kakatiya Institute of Technology and Science, Warangal - 506009. \\ E-mail: micharva@yahoo.com. \\ 2. Sreenidhi Institute of Science and Technology, Ghatkesar, Hyderabad-501 301
}

\begin{abstract}
Reaction of substituted 2-aminopyridines with diethylmalonates yields 2,4dihydroxy-1,8-Naphthyridenes(1-18). The 6-chloro-2,4-dihydroxy-1,8- naphthyridene (5) when treated with different reagents varied substituted derivatives are produced. 6chloro-2, 4-dihydroxy-1,8-naphthyridene(5) when treated with sodium azide offered 2,4-dihydroxy-1,8-naphthyridene-6-thiones (19-21). 6-azido-1,8-naphthyridine-2,4diols(22-24) were obtained by reacting 5 with sodiumazide. The 6-hydrazinyl-1,8naphthyridine-2,4-diols (25-27) and 2,4,6-trihydroxy-1,8-naphthyridenes (28-30) were produced by the reaction of 5 with hydrazine hydrate and acetic acid respectively.
\end{abstract}

Keywords: Naphthyridines, Thiazolidinones, Azitidinones.

\section{Introduction}

Naphthyridines attracted great attention of medical and synthetic chemists because of their versatile biological activity (1-6). Nalidixic acid, for example, possesses strong antibacterial activity and used mainly for the treatment of urinary tract infections with gram negative pathogens (7). Naphthyridine derivatives react with adenosine receptors of sub types $A_{1}$ and $A_{2} A(8)$. The major synthetic procedures to prepare 1,8naphthyridenes from 2 -aminopyridine with $\beta$-ketoesters (9). Keeping in mind the important properties of 1,8-naphthyridines, continuation of our earlier work on substituted 1-8-naphthyridines (10-14), we have synthesized a series of 2, 4dihydroxy-1, 8-Naphthyridenes without using solvent and catalyst from 2aminopyridine derivatives. The chloro group in 6-chloro-2,4-dihydroxy-1,8naphthyridene(5) was investigated with various hetero nucleophiles of the various reagents available for the replacement of chlorine by Sulphur, Sodiumsulphide was found to be an efficient reagent affording nucleophilic substitution by sulphur and also providing scope for further reaction (15-19). In this paper we describe the synthesis and biological activity of some 1, 8-naphthyridene derivatives.

\section{Results and Discussion}

2 -aminopyridine and diethylmalonate were irradiated in microwave oven at 400 watts to yield compound 1 . The same procedure is extended to prepare compounds 2-18. The compound $\mathbf{5}$ is treated to give different compounds by the substitution of $\mathbf{C l}$ group. In all cases the 'HNMR spectra include characterization of naphthyridine ring system. The activity of compounds was determined using cup-plate agar diffusion method by measuring the inhibition zone in $\mathrm{mm}$ and the data is given in table 1 . The characterization of the compounds is given in table 2 . 


\section{Scheme 1}

In conjunction with this work we report the synthesis of 1-18 by condensation of 2aminopyridines and diethylmalonates in which compound $\mathbf{8}$ was identical in all aspects with an authentic sample previously prepared (16) by normal procedure. Here, we prepared no. of compounds by microwave reaction.<smiles>[R3]c1c(N)nc(N)c([18O])c1[R]</smiles>

\section{Scheme 2}

Preparation of 19-21 is followed by substitution of $\mathrm{Cl}$ group by $\mathrm{SH}$ group using sodiumsulphide in compound 5 by microwave reaction (15).<smiles>[2H]c1c(O)nc2ncc(Cl)cc2c1O</smiles><smiles>[R]c1c(O)nc2ncc(S)cc2c1O</smiles>

$19 \mathrm{R}-\mathrm{H}, 20 . \mathrm{R}=\mathrm{Me}, 21 \mathrm{R}=\mathrm{CH}_{2}-\mathrm{Ph}$

\section{Scheme 3}

In order to construct new derivative of naphthyridines $22,23,24$ are obtained by the reaction of 5 and sodiumazide (15).<smiles>[2H]c1c(O)nc2ncc(Cl)cc2c1O</smiles><smiles>[R]c1c(O)nc2ncc(N)cc2c1O</smiles>

22. $\mathrm{R}=\mathrm{H}, 23 . \mathrm{R}=\mathrm{Me}, 24 \cdot \mathrm{R}=\mathrm{CH}_{2}-\mathrm{Ph}$

\section{Scheme 4}

The $\mathrm{Cl}$ group is next substituted by $\mathrm{NH}_{2} \mathrm{NH}_{2}$ group in compound 5 (15).

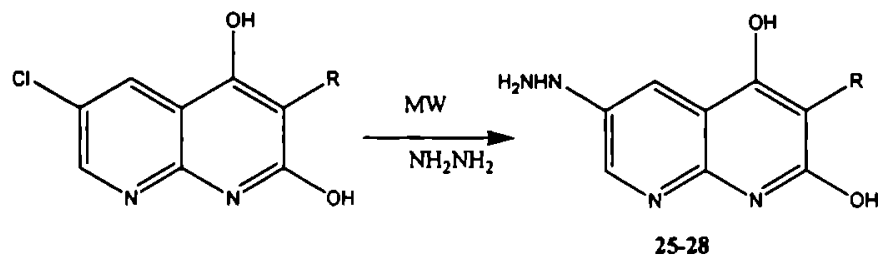




\section{Scheme 5}

Attention was next tuned to the substitution of $\mathrm{Cl}$ group by $\mathrm{OH}$ group in compound 5 . The reaction was catalysed by acetic acid (15).<smiles>Oc1cccc(N=Nc2c(O)nc3ncc(O)cc3c2O)c1</smiles>

\section{Experimental Section}

Melting points were measured in a Sulphuric acid bath and are uncorrected. The IR spectra were recorded on Brucher-IFS-66 FTIR instrument. $300 \mathrm{MHz}$ NMR spectra were recorded using tetramethyl silane as an instrumental standard in DMSO-d $\mathrm{d}_{6}$. Chemical shifts are expressed in ppm. The elemental analysis $(\mathrm{C}, \mathrm{N}, \mathrm{O})$ of these newly synthesized compounds was carried out t the purity of the compounds was checked by TLC and spots were visualized in iodine vapour.

General procedure for the preparation of 2-4-dihydroxy-1,8- naphthyridene derivatives (1-18) :

A mixture of 2-aminopyridene $(9.0 \mathrm{~m}$ mole $)$ and diethylmelonate $(10 \mathrm{~m}$ mole $)$ were ground well in a motor to get homogeneous mixture. This mixture was irradiated in micro wave oven at 400 watts for 3-4 min. The reaction mixture was taken out, cooled and digested with water. The products were recrystalised from ethanol.

Formation of 2,4-dihydroxy-4,8-naphthyridene-6-thiones : (19-21)

Compound ' 5 ' ( 1 mole), sodium sulphide( $1.5 \mathrm{~m}$ moles) were ground well to get lime powder and irradiated in microwave oven at 400 watts for 2 minutes. The reaction mixture was taken in to cold water and acidified with acetic acid. The solid obtained was filtered.

6-mercapto-1,8-naphthyridine-2,4-diol (19) ) IR: $3308.211 \mathrm{~cm}^{-1}(\mathrm{OH}), 1620.723 \mathrm{~cm}^{-1}$ $(\mathrm{C}=\mathrm{N}), 682.321 \mathrm{~cm}^{-1}(\mathrm{C}-\mathrm{S})$; ${ }^{1} \mathrm{HNMR}\left(\mathrm{DMSO}-\mathrm{d}_{6}\right) 7.31(\mathrm{~S}, 1 \mathrm{H}), 7.62(\mathrm{~S}, 1 \mathrm{H}), 7.91(\mathrm{~S}$, 1H) 8.52 (brs, 2H), $11.82(\mathrm{~S}, 1 \mathrm{H})$.

6-mercapto-3-methyl-1,8-naphthyridine-2,4-diol (20) 'HNMR (DMSO-d $\mathrm{d}_{6}$ ) 2.31 (S, $1 \mathrm{H}), 7.61(\mathrm{~S}, 1 \mathrm{H}), 7.92(\mathrm{~S}, 1 \mathrm{H}) 8.45(\mathrm{brs}, 2 \mathrm{H}), 11.78(\mathrm{~S}, 1 \mathrm{H})$.

3-benzyl-6-mercapto-1,8-naphthyridine-2,4-diol (21) 'HNMR (DMSO-d 6 ) 4.24 (dd, 2H, J=12.08 Hz), 7.18-7.35 (M, 5H), 7.61 (S, 1H) 7.93 (S, 1H), 8.47 (brs, 2H) 11.77 $(\mathrm{S}, 1 \mathrm{H})$.

\section{Formation of 6-azido-1,8-naphthyridine-2,4-diols : (22-24)}

Compound $5(0.01 \mathrm{mmole})$ a pinch of PTSA and sodium azide $(0.01 \mathrm{mmol})$ were taken in to a beaker and ground well to get power. The contents are irradiated in micro oven at 400 watts for $3-4 \mathrm{~min}$. The reaction mixture was washed with water and the solid obtained is purified by recrystalisation. 
6-azido-1,8-naphthyridine-2,4-diol (22) IR: $3018.122 \mathrm{~cm}^{-1}$ (C-H aromatic), 2122.231 $\mathrm{cm}^{-1}\left(\mathrm{~N}_{3}\right), 1616.412 \mathrm{~cm}^{-1}(\mathrm{C}=\mathrm{N}){ }^{\prime}$ HNMR (DMSO-d 6 ) $7.32(\mathrm{~S}, 1 \mathrm{H}), 7.85(\mathrm{~S}, 1 \mathrm{H}) 8.30$ $(\mathrm{S}, 1 \mathrm{H}), 8.55$ (brs, 2H).

6-azido-3-methyl-1,8-naphthyridine-2,4-diol (23) ${ }^{1} \mathrm{HNMR}\left(\right.$ DMSO-d $\left._{6}\right) 2.312(\mathrm{~S}, 3 \mathrm{H})$, $7.84(\mathrm{~S}, 1 \mathrm{H}) 8.28(\mathrm{~S}, 1 \mathrm{H}), 8.48$ (brs, $2 \mathrm{H})$.

6-azido-3-benzyl-1,8-naphthyridine-2,4-diol (24) ${ }^{1} \mathrm{HNMR}$ (DMSO-d 6 ) 4.12 (dd, 2H, J $=12.08 \mathrm{~Hz}), 7.15-7.31(\mathrm{~m}, 5 \mathrm{H}) 7.85(\mathrm{~S}, 1 \mathrm{H}), 8.29(\mathrm{~S}, 1 \mathrm{H}), 8.45(\mathrm{brs}, 2 \mathrm{H})$.

Formation of 6-hydrazinyl-1,8-naphthyridine-2,4-diols (25-27)

To a solution of $5(0.01 \mathrm{mmol})$ and hydrazine hydrate $(0.01 \mathrm{mmole})$ and a pinch of sodium acetate are intimately mixed and irradiated in MW oven for 3-4 min. products obtained were washed with cold water and purified by recrystalisation.

6-hydrazinyl-1,8-naphthyridine-2,4-diol (25) IR: $3458.148 \mathrm{~cm}^{-1}(\mathrm{~N}-\mathrm{H}), 3022.241 \mathrm{~cm}^{-1}$ (C-H aromatic), $1616.422 \mathrm{~cm}^{-1}$ (C=N); 'HNMR (DMSO-d ${ }_{6}$ ) 4.41 (brs, 3H), 7.28 (S, $1 \mathrm{H}), 7.58(\mathrm{~S}, 1 \mathrm{H}), 8.21(\mathrm{~S}, 1 \mathrm{H}) 8.32$ (brs, 2H).

6-hydrazinyl-3-methyl-1,8-naphthyridine-2,4-diol (26) ${ }^{1} \mathrm{HNMR}$ (DMSO-d 6 ) 2.31 (S, $3 \mathrm{H}), 3.82$ (brs, 3H), 7.29 (S, 1H), 8.22 (S, 1H) 8.38 (brs, 2H).

3-benzyl-6-hydrazinyl-1,8-naphthyridine-2,4-diol (27) ' HNMR (DMSO-d 6 ) 3.75 (brs, $3 \mathrm{H}), 4.28(\mathrm{dd}, 2 \mathrm{H}, \mathrm{J}=12.08 \mathrm{~Hz}) 7.11-7.28(\mathrm{~m}, 5 \mathrm{H}), 7.30(\mathrm{~S}, 1 \mathrm{H}), 8.25(\mathrm{~S}, 1 \mathrm{H}) 8.40$ (brs, 2H).

\section{Synthesis of 2,4,6-trihydroxy-1,8-naphthyridene (28-30)}

Compound ' 5 ' $(1 \mathrm{~m}$ mole $)$ and in $70 \%$ acetic and $(10 \mathrm{ml})$ was heated under reflux for 4-6 hrs. The completion of reaction was chelcked by TLC upon cooling the reaction mixture a solid product precipitated out, which was filtered washed well with water.

1,8-naphthyridine-2,4,6-triol (28) ) IR: $3310.235 \mathrm{~cm}^{-1}(\mathrm{O}-\mathrm{H}), 1618.124 \mathrm{~cm}^{-1}(\mathrm{C}=\mathrm{N})$; ${ }^{1} \mathrm{HNMR}\left(\mathrm{DMSO}-\mathrm{d}_{6}\right) 7.28(\mathrm{~S}, 1 \mathrm{H}), 7.62(\mathrm{~S}, 1 \mathrm{H}), 7.98(\mathrm{~S}, 1 \mathrm{H}) 8.30$ (brs, 3H)

3-methyl-1,8-naphthyridine-2,4,6-triol (29) 'HNMR (DMSO-d $\mathrm{d}_{6}$ ) 2.31 (s, 3H), 7.63 (S, $1 \mathrm{H}), 7.99(\mathrm{~S}, 1 \mathrm{H}), 8.32$ (brs, 3H)

3-benzyl-1, 8-naphthyridine-2, 4,6-triol (30) ${ }^{1} \mathrm{HNMR}$ (DMSO-d 6 ) 4.12 (dd, 2H, 12.08 $\mathrm{Hz}), 7.62(\mathrm{~S}, 1 \mathrm{H}), 7.98(\mathrm{~S}, 1 \mathrm{H}), 8.38(\mathrm{brs}, 3 \mathrm{H}), 7.12-7.32$ (m, 5H). 
Table 1

ANTIBACTERIAL ACTIVITY OF COMPOUNDS AGAINST GRAM +VE AND GRAM -VE BACTERIA

\begin{tabular}{|c|c|c|c|c|c|c|c|}
\hline $\begin{array}{c}\text { S.N } \\
\text { o. }\end{array}$ & $\begin{array}{c}\text { Compn } \\
\mathbf{d}\end{array}$ & $\begin{array}{c}\text { Staphyl } \\
\text { ococcus } \\
\text { aureus }\end{array}$ & $\begin{array}{c}\text { Klebsiella } \\
\text { pneumoniae }\end{array}$ & $\begin{array}{c}\text { Bacillus } \\
\text { cereus }\end{array}$ & $\begin{array}{c}\text { Pseudomonas } \\
\text { putida }\end{array}$ & $\begin{array}{c}\text { Salmone } \\
\text { Ila } \\
\text { paratyph } \\
\text { iA }\end{array}$ & $\begin{array}{c}\text { Salmonell } \\
\text { a } \\
\text { paratyphi } \\
\text { B }\end{array}$ \\
\hline 1 & 1 & 1 & 3 & 1 & - & 4 & 1 \\
\hline 2. & 5 & 4 & 3 & 2 & 2 & 2 & 6 \\
\hline 3. & 11 & 5 & 3 & 2 & 1 & 2 & 6 \\
\hline 4. & 17 & 6 & 3 & 1 & 1 & 2 & 6 \\
\hline 5. & 19 & 6 & 5 & 3 & 6 & 2 & - \\
\hline 6. & 20 & 6 & 4 & 4 & 6 & 2 & 1 \\
\hline 7. & 21 & 7 & 5 & 5 & 6 & 1 & 2 \\
\hline 8. & 22 & 1.5 & - & 9 & 2 & 3 & - \\
\hline 9. & 23 & 2 & - & 9 & 3 & 4 & - \\
\hline 10. & 24 & 4 & 2 & 10 & 3 & 5 & 1 \\
\hline 11. & 25 & 9 & 2 & 1 & 5 & 5 & 1 \\
\hline 12. & 26 & 10 & 3 & 1 & 5 & 4 & 2 \\
\hline 13. & 27 & 12 & 4 & 2 & 7 & 7 & 1 \\
\hline 14. & 28 & 5 & - & 6 & 5 & 3 & - \\
\hline 15. & 29 & 5 & 1 & 7 & 7 & 4 & 1 \\
\hline 16. & 30 & 7 & 1 & 9 & 9 & 5 & 2 \\
\hline
\end{tabular}

INHIBITION ZONE IN MM (- indicates no inhibitory activity)

Control inhibition zone (which indicates inhibition zone of solvent) was subtracted from inhibition zone of compounds which gives actual inhibition zone of compounds

1.Staphylococcus aureus - coccus, gram +ve,causes toxic shock syndrome(TSS).

2. Klebsiella pneumoniae -rod shape or bacilli,gram-ve,causes pneumonia

3. Bacillus cereus - rod ,gram +ve,causes food borne diseases

4. Pseudomonas putida - rod,gram -ve

5. Salmonella paratyphi $A$ - rod,gram-ve,causes typhoid

6. Salmonella paratyphi $B$ - rod,gram -ve,causes typhoid

Perusal of the above table reveals that the derivatives having chlorine as substituent is more toxic than simple dihydroxy compound to all six bactereas. Among the chloro group compounds, the compound which has methyl group and benzyl group slight more toxic than 5-chloro, 2-4 dihydroxy 1,8 Naphthyridine. Azido substituent has shown toxicity to bactereas except Salmonella paratyphi B and Klebsiella pneumonia. Thio and hydroxyl substituted derivatives are also more toxic towards all bacterias. The compounds which have hydrazine substituent have shown versatile toxicity to all bactereas 
Table 2

Characterization table of 2,4-Dihydroxy 1,8 - Naphthyridines

\begin{tabular}{|c|c|c|c|c|c|c|c|}
\hline Comnd & $\mathbf{R}$ & $\mathbf{R}_{\mathbf{1}}$ & $\mathbf{R}_{\mathbf{2}}$ & $\mathbf{R}_{\mathbf{3}}$ & M.Formula & $\underset{{ }^{\circ} \mathrm{C}}{\text { M.P }}$ & $\begin{array}{c}\text { Mass } \\
(\mathbf{M}+1)\end{array}$ \\
\hline 1. & $\mathrm{H}$ & $\mathrm{H}$ & $\mathrm{H}$ & $\mathrm{H}$ & $\mathrm{C}_{8} \mathrm{H}_{6} \mathrm{~N}_{2} \mathrm{O}$ & $268-270$ & 163 \\
\hline 2. & $\mathrm{H}$ & $\mathrm{H}$ & $\mathrm{H}$ & $\mathrm{Me}$ & $\mathrm{C}_{8} \mathrm{H}_{7} \mathrm{~N}_{3} \mathrm{O}$ & $>300$ & 177 \\
\hline 3. & $\mathrm{H}$ & $\overline{\mathrm{H}}$ & $\overline{\mathrm{H}}$ & $\mathrm{NH}_{2}$ & $\mathrm{C}_{9} \mathrm{H}_{8} \mathrm{~N}_{2} \mathrm{O}$ & $278-280$ & 178 \\
\hline 4. & $\mathrm{H}$ & $\mathrm{H}$ & $\mathbf{M e}$ & $\mathrm{H}$ & $\mathrm{C}_{9} \mathrm{H}_{8} \mathrm{~N}_{2} \mathrm{O}$ & $265-268$ & 177 \\
\hline 5. & $\mathrm{H}$ & $\mathrm{H}$ & $\mathrm{Cl}$ & $\mathrm{H}$ & $\mathrm{C}_{9} \mathrm{H}_{8} \mathrm{~N}_{2} \mathrm{O}$ & $267-270$ & 197 \\
\hline 6. & $\overline{\mathrm{H}}$ & $\mathrm{Me}$ & $\mathrm{H}$ & $\mathrm{H}$ & $\mathrm{C}_{8} \mathrm{H}_{3} \mathrm{ClN}_{2} \mathrm{O}$ & $270-272$ & 177 \\
\hline 7. & $\mathrm{Me}$ & $\mathrm{H}$ & $\mathrm{H}$ & $\mathrm{H}$ & $\mathrm{C}_{9} \mathrm{H}_{8} \mathrm{~N}_{2} \mathrm{O}_{2}$ & $270-272$ & 177 \\
\hline 8. & $\mathrm{Me}$ & $\mathrm{H}$ & $\mathrm{H}$ & $\mathrm{Me}$ & $\mathrm{C}_{10} \mathrm{H}_{10} \mathrm{~N}_{2} \mathrm{O}_{2}$ & $>300$ & 191 \\
\hline 9. & $\mathrm{Me}$ & $\mathrm{H}$ & $\mathrm{H}$ & $\mathrm{NH}_{2}$ & $\mathrm{C}_{9} \mathrm{H}_{7} \mathrm{~N}_{2} \mathrm{O}_{2} \mathrm{Cl}$ & $>300$ & 192 \\
\hline 10. & $\mathrm{Me}$ & $\mathrm{H}$ & $\mathrm{Me}$ & $\mathrm{H}$ & $\mathrm{C}_{10} \mathrm{H}_{10} \mathrm{~N}_{2} \mathrm{O}_{2}$ & $>300$ & 191 \\
\hline 11. & $\mathrm{Me}$ & $\mathrm{H}$ & $\mathrm{Cl}$ & $\mathrm{H}$ & $\mathrm{C}_{15} \mathrm{H}_{12} \mathrm{~N}_{2} \mathrm{O}_{2}$ & $290-292$ & 211 \\
\hline 12. & $\mathbf{M e}$ & $\mathrm{Me}$ & $\mathrm{H}$ & $\mathrm{H}$ & $\mathrm{C}_{16} \mathrm{H}_{14} \mathrm{~N}_{2} \mathrm{O}_{2}$ & $>300$ & 191 \\
\hline 13. & $\mathrm{CH}_{2} \mathrm{Ph}$ & $\mathrm{H}$ & $\mathrm{H}$ & $\mathrm{H}$ & $\mathrm{C}_{15} \mathrm{H}_{13} \mathrm{~N}_{3} \mathrm{O}_{2}$ & $260-263$ & 253 \\
\hline 14. & $\mathrm{CH}_{2} \mathrm{Ph}$ & $\mathrm{H}$ & $\mathrm{H}$ & $\mathrm{Me}$ & $\mathrm{C}_{16} \mathrm{H}_{14} \mathrm{~N}_{2} \mathrm{O}_{2}$ & $267-270$ & 268 \\
\hline 15. & $\mathrm{CH}_{2} \mathrm{Ph}$ & $\mathrm{H}$ & $\mathrm{H}$ & $\mathrm{NH}_{2}$ & $\mathrm{C}_{16} \mathrm{H}_{11} \mathrm{~N}_{2} \mathrm{O}_{2} \mathrm{Cl}$ & 258-262 & 269 \\
\hline 16. & $\mathrm{CH}_{2} \mathrm{Ph}$ & $\mathrm{H}$ & $\mathrm{Me}$ & $\mathrm{H}$ & $\mathrm{C}_{16} \mathrm{H}_{14} \mathrm{~N}_{2} \mathrm{O}_{2}$ & $266-268$ & 268 \\
\hline 17. & $\mathrm{CH}_{2} \mathrm{Ph}$ & $\overline{\mathrm{H}}$ & $\mathrm{Cl}$ & $\overline{\mathrm{H}}$ & $\mathrm{C}_{6} \mathrm{H}_{11} \mathrm{~N}_{2} \mathrm{O}_{2} \mathrm{Cl}$ & $272-274$ & 289.5 \\
\hline 18. & $\mathrm{CH}_{2} \mathrm{Ph}$ & $\mathrm{Me}$ & $\mathrm{H}$ & $\mathrm{H}$ & $\mathrm{C}_{16} \mathrm{H}_{14} \mathrm{~N}_{2} \mathrm{O}_{2}$ & 266-268 & 2.68 \\
\hline 19. & $\mathrm{H}$ & - & - & - & $\mathrm{C}_{8} \mathrm{H}_{6} \mathrm{~N}_{2} \mathrm{O}_{2} \mathrm{~S}$ & $256-258$ & 195 \\
\hline 20. & $\mathrm{Me}$ & - & - & - & $\mathrm{C}_{9} \mathrm{H}_{8} \mathrm{~N}_{2} \mathrm{O}_{2} \mathrm{~S}$ & $260-262$ & 209 \\
\hline 21. & $\mathrm{CH}_{2} \mathrm{Ph}$ & - & - & - & $\mathrm{C}_{15} \mathrm{H}_{12} \mathrm{~N}_{2} \mathrm{O}_{2} \mathrm{~S}$ & $261-263$ & 285 \\
\hline 22. & $\mathrm{H}$ & - & - & - & $\mathrm{C}_{8} \mathrm{H}_{5} \mathrm{~N}_{3} \mathrm{O}_{2}$ & $258-260$ & 204 \\
\hline 23. & $\mathrm{Me}$ & - & - & - & $\mathrm{C}_{9} \mathrm{H}_{7} \mathrm{~N}_{3} \mathrm{O}_{2}$ & $262-264$ & 218.5 \\
\hline 24. & $\mathrm{CH}_{2} \mathrm{Ph}$ & - & - & - & $\mathrm{C}_{15} \mathrm{H}_{4} \mathrm{~N}_{5} \mathrm{O}_{2}$ & $262-264$ & 294.1 \\
\hline 25. & $\mathrm{H}$ & - & - & - & $\mathrm{C}_{8} \mathrm{H}_{8} \mathrm{~N}_{4} \mathrm{O}_{2}$ & $256-258$ & 193.2 \\
\hline 26. & $\mathrm{Me}$ & - & - & - & $\mathrm{C}_{9} \mathrm{H}_{10} \mathrm{~N}_{4} \mathrm{O}_{2}$ & $258-260$ & 207.1 \\
\hline 27. & $\mathrm{CH}_{2} \mathrm{Ph}$ & - & - & $=$ & $\mathrm{C}_{15} \mathrm{H}_{14} \mathrm{~N}_{4} \mathrm{O}_{2}$ & $254-256$ & 283.3 \\
\hline 28. & $\mathrm{H}$ & - & - & $=$ & $\mathrm{C}_{8} \mathrm{H}_{6} \mathrm{~N}_{2} \mathrm{O}_{3}$ & 264-268 & 179.1 \\
\hline 29. & $\mathrm{Me}$ & - & - & - & $\mathrm{C}_{9} \mathrm{H}_{8} \mathrm{~N}_{2} \mathrm{O}_{3}$ & $268-271$ & 193.2 \\
\hline 30. & $\mathrm{CH}_{2} \mathrm{Ph}$ & - & - & - & $\mathrm{C}_{15} \mathrm{H}_{11} \mathrm{~N}_{2} \mathrm{O}_{3}$ & $269-272$ & 269.1 \\
\hline
\end{tabular}

\section{Acknowledgements}

Authors are thankful to management, Director, Principal and Head, Department of Bio-Technology Head, Department of Science and Humanities of SNIST \& Management and Principal of KITS for providing research facilities, for their grants and for their encouragement. 


\section{References}

(1). H. Egawa. A.Miyamido.; Y. Nishimra.; H. Okada.; Uno H., Matsumato, J. J Med. Chem. 27, 1543 (1984).

(2). J .Nezval; J.Halocka Experientia 23, 1043 (1967).

(3). E. M. Hawes, J. Med. Chem., , 20, 124 (1977).

(4). N. Suzuki. Chem. Pharma. Bull., , 28, 761 (1980).

(5). N. Suzuki and R. Dohmori. Chem. Pharma. Bull.. 27, 410 (1979).

(6). G. B. Balin and W. L Tan. Aust. J.Chem., 37, 1065 (1984).

(7). P.M.Gilis,; A.Haemers; W.Bollaert, J, Heterocycl. Chem., 17, 717 (1980).

(8). C.J.Muller; B.Grahner; D.Heber, pharmazie, 49, 878 (1994).

(9). P.L,Ferrarini,; C.Mori, J. Heterocucl. Chem., 27, 881 (1990).

(10). A. Da Settimo.; G. Biagi.; G. Primofiore.; P. L Ferarini.; O.Livi and A. M. Marini. J. Heterocycl. Chem, 17, 1225 (1980).

(11). M.Thirumala Chary; K.Mogilaiah; B.Sreenivasulu J. Indian Chem. Soc. 64, 488 (1987).

(12). K. Mogilaiah.; K. Vijayender Reddy and B. Sreenivasulu. Indian J. Chem., Sect., 22, 178 (1983).

(13). G. R. Rao.; K Mogilaiah and B. Sreenivasulu. Collect Czech. Chem. Commun., 54, 254 (1989).

(14). M T. Chary.; K. Mogilaiah.; B. Swamy \& B. Sreenivasulu. Sulfur Left , , 8, 79 (1988).

(15). R.M Singh, Indian Journal of Chemistry 44B 1868-1875 (2005).

(16). Muwaffag.; Manera.; Mori.; Sccomanni and Ferrarini. II Farmaco, , 57, 631 (2002).

(17). Venugopal.; Perumal and Rajadurari. Tetrahedron let, , 913 (1974).

(18). LB.Young; and WS.Trahanovsky J Org Chem, 31, 2033 91966).

(19). Ramadan, A.Mekheimer.; Afaf M. Abdel Hameed.; Kamal U. Sadek. Arkivoc xiii 269 (2007).

Received on July 3, 2008. 
